\title{
IncRNA co-expression network model for the prognostic analysis of acute myeloid leukemia
}

\author{
JIA-QI PAN ${ }^{*}$, YAN-QING ZHANG ${ }^{*}$, JING-HUA WANG, PING XU and WEI WANG \\ Department of Hematology, The Second Affiliated Hospital of \\ Harbin Medical University, Harbin, Heilongjiang 150001, P.R. China
}

Received February 23, 2016; Accepted February 2, 2017

DOI: $10.3892 /$ ijmm.2017.2888

\begin{abstract}
Acute myeloid leukemia (AML) is a highly heterogeneous hematologic malignancy with great variability of prognostic behaviors. Previous studies have reported that long non-coding RNAs (lncRNAs) play an important role in AML and may thus be used as potential prognostic biomarkers. However, thus use of lncRNAs as prognostic biomarkers in AML and their detailed mechanisms of action in this disease have not yet been well characterized. For this purpose, in the present study, the expression levels of lncRNAs and mRNAs were calculated using the RNA-seq V2 data for AML, following which a lncRNA-lncRNA co-expression network (LLCN) was constructed. This revealed a total of 8 AML prognosis-related lncRNA modules were identified, which displayed a significant correlation with patient survival ( $\mathrm{p} \leq 0.05)$. Subsequently, a prognosis-related lncRNA module pathway network was constructed to interpret the functional mechanism of the prognostic modules in AML. The results indicated that these prognostic modules were involved in the AML pathway, chemokine signaling pathway and WNT signaling pathway, all of which play important roles in AML. Furthermore, the investigation of lncRNAs in these prognostic modules suggested that an lncRNA (ZNF571-AS1) may be involved in AML via the Janus kinase (JAK)/signal transducer and activator of transcription (STAT) signaling pathway by regulating KIT and STAT5. The results of the present study not only provide potential lncRNA modules as prognostic biomarkers, but also provide further insight into the molecular mechanisms of action of lncRNAs.
\end{abstract}

Correspondence to: Dr Wei Wang, Department of Hematology, The Second Affiliated Hospital of Harbin Medical University, Harbin, Heilongjiang 150001, P.R. China

E-mail: wangweihmu@126.com

${ }^{*}$ Contributed equally

Key words: long non-coding RNA, acute myeloid leukemia, network

\section{Introduction}

Acute myeloid leukemia (AML) is a highly heterogeneous hematologic malignancy with great variability in biological, phenotypic and prognostic behaviors and strikingly diverse outcomes to standard therapy $(1,2)$. Despite significant advances in the treatment of patients with AML, the prognosis of many patients is still uncertain and optimal post-remission therapy is unclear. Thus, the elucidation of AML survival events is important and may potentially aid in the prognosis and treatment of patients with AML.

In recent years, a number of studies on prognostic markers in AML have focused on non-coding RNAs (ncRNAs), which lack protein-coding potential. The majority of these studies have focused on miRNAs (3-6). However, there are few reports on long non-coding RNA (lncRNA) as prognostic markers in AML. IncRNAs are transcripts which are $>200$ nucleotides in length, located within intergenic stretches or overlapping antisense transcripts of protein-coding genes. They have emerged as important regulators of gene expression, showing cell-specific expression patterns and subcellular localization and are involved in many biological functions, including cell apoptosis, proliferation and the cell cycle $(7,8)$. Some studies have demonstratd that lncRNAs are associated with AML. For example, lncRNA CCD26 has been shown to control the growth of myeloid leukemia cells through the regulation of KIT expression (9). Thus, it is reasonable that lncRNAs may be considered as prognostic biomarkers.

ncRNAs rarely function in isolation, but always function together to form biological modules (10). These functional biological modules are often considered to be prognostic biomarkers due to their improved robustness and interpretability (11). A number of methods have been developed to discover functional modules, such as weighted gene co-expression network analysis (WGCNA). WGCNA is a systems biology-based approach, which offers a promising technique for detecting functional modules (12). WGCNA has been widely used to identify functional modules that contribute to phenotypic traits in various diseases (13-17). Compared with other techniques based on gene expression profiling network analysis, such as cytoscape-based approaches, WGCNA transforms gene expression profiles into functional co-expressed gene modules, which do not rely on prior assumptions about genes or covariates, thereby providing insight into biological 
signaling networks that may be associated with phenotypic traits of interest (18). In this study, we used WGCNA to identify lncRNA co-expression modules.

The Cancer Genome Atlas (TCGA) stores comprehensive datasets of multiple cancers, including clinical data and transcriptome data of AML. The expression levels of lncRNAs and mRNAs in AML were calculated using RNA-seq V2 dataset. There is evidence to indicate that IncRNAs may play a functional role by regulating gene expression, predominantly by their secondary structures, which is difficult to decipher (19). Considering the challenges in investigating the functional mechanisms of lncRNA modules, a co-expression mRNA-based method was used in this study, in which the functions of IncRNA modules were predicted according to their co-expressed protein-coding gene (19).

In this study, to identify prognosis-related lncRNA modules and the potential mechanisms of AML, the expression of lncRNAs was calculated using the RNA-seq V2 dataset of TCGA and an AML-related lncRNA co-expression network was constructed. Subsequently, WGCNA was used to identify AML functional lncRNA co-expression modules. Based on survival analysis, 8 prognosis-related lncRNA modules for AML were identified. Module 27 was the most significant prognosis-related lncRNA module, which displayed the best performance in the survival prediction (log-rank test, $\mathrm{p}=0.000502$ ). To investigate the mechanisms of action of these prognosis-related lncRNA modules, pathway enrichment of all co-expressed mRNAs of lncRNA modules was implemented, and a prognosis module-pathway network was constructed to interpret the mechanisms of AML. The results of the present study not only provide potential lncRNA modules as prognostic biomarkers, but also provide further insight into the molecular mechanisms of action of lncRNAs.

\section{Materials and methods}

Data. The RNA-seq data set of AML was downloaded from TCGA (https://tcga-data.nci.nih.gov/). This dataset was derived from the tissue samples of 200 adult patients with de novo AML using RNA-seq technology. The clinical survival data was also obtained from TCGA. Survival time was defined as the time from tissue removal to death, lossto-follow-up or study conclusion. Patients who were lost to follow-up or survival time after $<20$ days were deleted from the next survival analysis. Finally, a total of 161 clinical samples remained in this study.

Expression of IncRNAs and mRNAs in AML. The RNA-seq V2 dataset of AML data was downloaded from the TCGA database, with quantile-normalized and background-corrected at level 3. The reads per kilobases per million reads (RPKM) values of genes and IncRNAs were calculated from exon read counts data, with $\mathrm{RPKM}=\left(\right.$ raw read counts $\left.\times 10^{6}\right) /($ total reads $x$ length of lncRNA/gene), in which, the raw read counts represented all exon read counts that mapped into a certain lncRNA/gene, and total reads were all exon read counts that mapped into all lncRNAs/genes of one single sample.

Construction of IncRNA-lncRNA co-expression network. The expression values of lncRNAs were obtained as described above. Next, if the missing rate of lncRNA or mRNA expression was $>90 \%$, the AML patients were excluded from this study. Finally, we obtained 1,406 lncRNAs across 173 AML patients. Pearson's correlation coefficient (PCC) and significant $\mathrm{p}$-value were calculated between the expression values of each lncRNA-lncRNA pair across all AML samples. The IncRNA-lncRNA pairs with a p-value $<0.001$ and absolute value of PCC $>0.5$ were used to construct a lncRNA-IncRNA co-expression network (LLCN). Finally, the LLCN was constructed with IncRNAs nodes, which were connected based on the significance of co-expression between them (Fig. 2).

Identification of prognosis-related lncRNA modules for $A M L$. Following the construction of the LLCN, the IncRNA co-expression modules were identified by a WGCNA R-based package, which could extract functional modules based on pairwise correlated expression among lncRNAs with co-regulation implications. Prior to the identification of lncRNA co-expression modules using WGCNA, sample clustering was first used to detect outliers. As a result, one sample identified as an outlier was excluded from the analysis (data not shown). In this study, we defined the prognosis-related lncRNA modules for AML as these modules are significantly related to the survival time of AML patients. To identify prognosisrelated IncRNA modules, the lncRNAs were extracted from each lncRNA co-expression module as signatures to perform survival analysis. Firstly, AML samples were divided into 2 groups based on the expression values of signature lncRNAs for each lncRNA co-expression module using a K-mean clustering method (20). The survival differences between 2 groups were then assessed by Kaplan-Meier estimate and compared using a log-rank test, where a p-value $<0.05$ was considered to indicate a significant result. The lncRNA co-expression module was considered to be a prognosis-related lncRNA module.

Functional analysis of AML-related lncRNA modules. To better illustrate the potential functional mechanism of prognosis-related lncRNA modules of AML, the Kyoto encyclopedia of genes and genomes (KEGG) pathway and gene ontology (GO) functional enrichment analyses were carried out using the database for annotation, visualization and integrated discovery (DAVID) (21), which consists of an integrated biological knowledge base and analytic tools aimed at systematically extracting biological meaning from large gene/protein lists. To do this, firstly, co-expression correlation and significance were calculated with PCC between the expression values of mRNA and lncRNA in prognosis-related lncRNA module across all matched AML samples. Subsequently, for each prognosis-related lncRNA module, the mRNA with Pearson's correlation test $\mathrm{p}$-value $<0.001$ and absolute value of $\mathrm{PCC}>0.5$ were used for functional enrichment analysis to investigate the mechanism of this module in AML.

\section{Results}

In this study, we identified AML prognosis-related lncRNA co-expression modules and interpreted their functional mechanisms in AML. The framework of this study is shown in Fig. 1. Firstly, we obtained clinical data and RNA-seq V2 


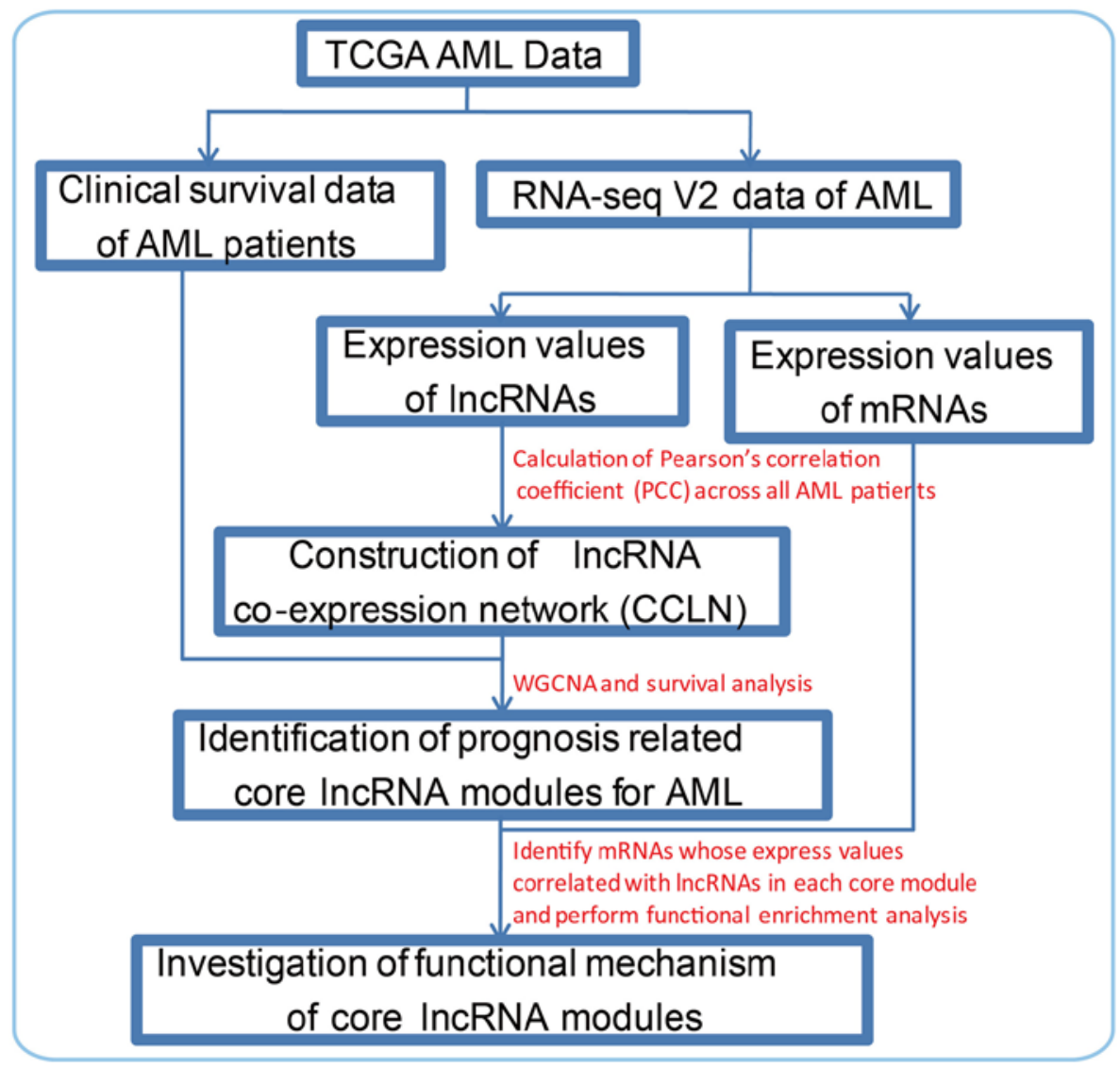

Figure 1. Flow chart of the procedures used in the present study.

data from TCGA. The expression values of lncRNAs and mRNAs in all AML patients were calculated. Subsequently, a lncRNA co-expression network was constructed by calculating co-expression PCC. To identify prognosis-related lncRNA modules for AML, clinical survival data were also used to identify lncRNA modules which are significantly associated with the survival time of AML patients. Lastly, we performed functional enrichment analysis using mRNA with expression values correlated with lncRNAs in each prognosis-related lncRNA module to interpret the mechanisms of AML.

Construction of $L L C N$. Based on co-expression correlations identified between each IncRNA-IncRNA pair across all AML samples with lncRNA-lncRNA pairs with a p-value $<0.001$ and an absolute value of PCC $>0.5$, the LLCN was constructed. The LLCN contained a total of 1,870 lncRNAs with 83,135 edges between them (Fig. 2) (data not shown). The degree of distribution of IncRNA nodes of LLCN followed power law distribution with correlations of 0.910 and $\mathrm{R}^{2}=0.877$, lncRNA RP11-492A10.1 with the highest degree.

Identification of prognosis-related lncRNA modules for AML. To identify prognosis-related lncRNA modules for AML, firstly, we identified functional lncRNA co-expression modules based on LLCN using WGCNA. A total of 42 co-expression lncRNA modules were identified, the largest module contained 427 lncRNAs and the smallest module contained 7 lncRNAs, with an average 44.7 lncRNAs per co-expression module (data not shown). Subsequently, for each co-expression lncRNA module, we performed survival analysis to determine whether it is a prognosis-related lncRNA module. In this process, AML patients were divided into 2 risk groups according to expression values of all lncRNAs contained in each lncRNA co-expression module in the IncRNA expression profile, and p-value calculations were based on log-rank test (Materials and methods). IncRNA co-expression modules with a p-value $<0.05$ were considered as prognosis-related lncRNA modules. Finally, we obtained 8 prognosis-related lncRNA modules from 42 lncRNA co-expression modules (Figs. 2 and 3). As shown in Table I, the largest prognosis-related lncRNA module contained 88 lncRNAs (module 3 ) and the smallest contained 7 lncRNAs (module 41). Module 27 is the most significant prognosis-related lncRNA module, which displayed the optimal performance in the survival prediction (log-rank test $\mathrm{p}=0.000502$ ). This module consisted of 17 lncRNAs; module 29 was the second most significant prognosis-related IncRNA module (log-rank test $\mathrm{p}=0.000779)$, which contained 16 lncRNAs (Fig. 2 and Table I).

Functional analyses of IncRNA modules in AML. To further investigate the mechanisms of action of prognosis-related lncRNA modules in AML, we performed functional pathway enrichment analysis using co-expression genes of lncRNAs in each lncRNA module. We found that 8 prognosis-related lncRNA modules wee significantly enriched in 70 pathways, which included certain AML-related pathways, such as the acute myeloid leukemia pathway, chemokine signaling pathway (22), pathway in cancer, as well as others. Subsequently, 
Module 29

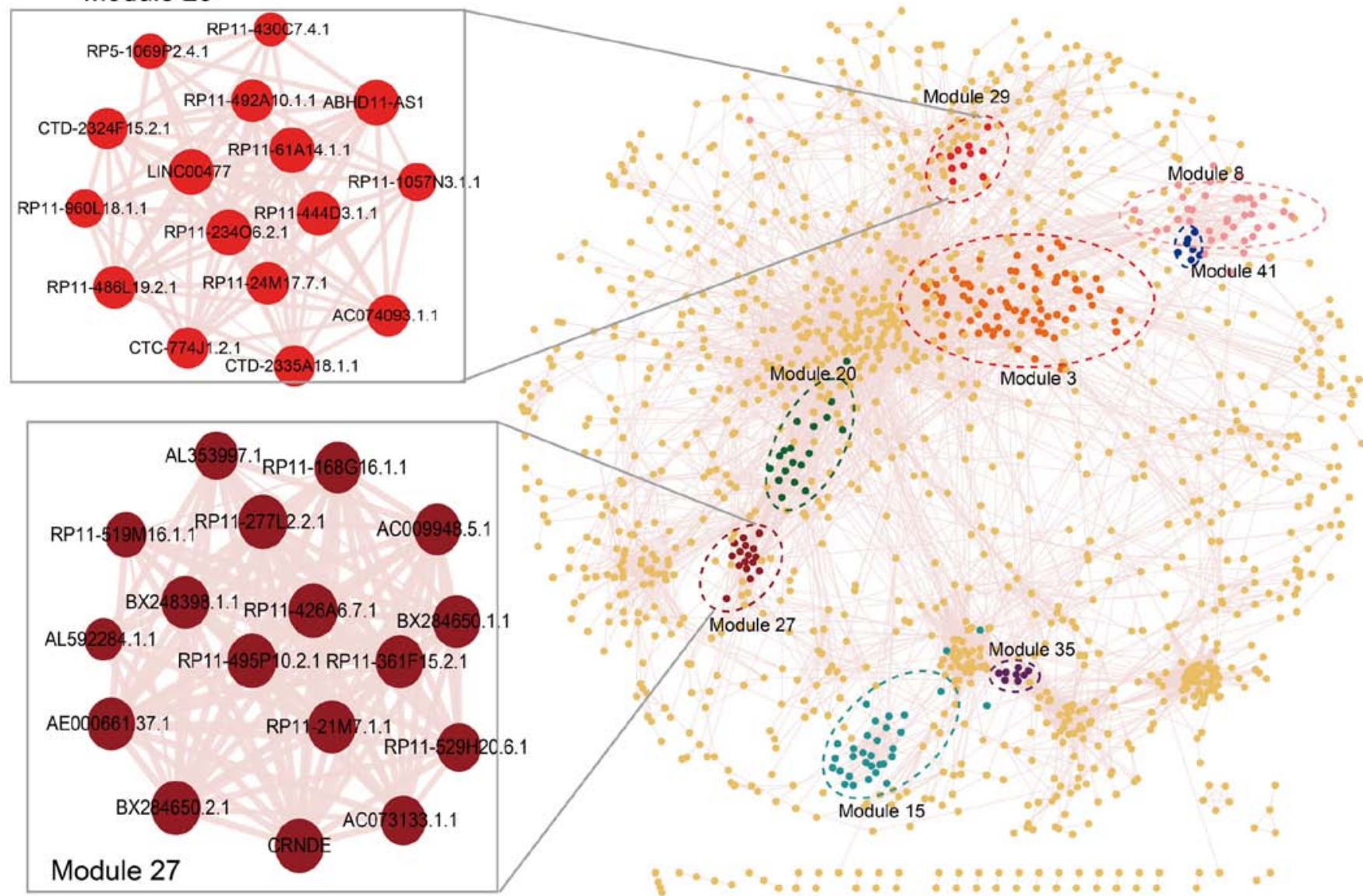

Figure 2. Long non-coding RNA (lncRNA)-lncRNA co-expression network (LLCN) of acute myeloid leukemia (AML) and prognosis-related lncRNA co-expression modules. The circles represent nodes of lncRNAs. The nodes in dotted circles in different colors represent different modules. The most significant prognosis modules, modules 27 and 29 are zoomed in (left panels).
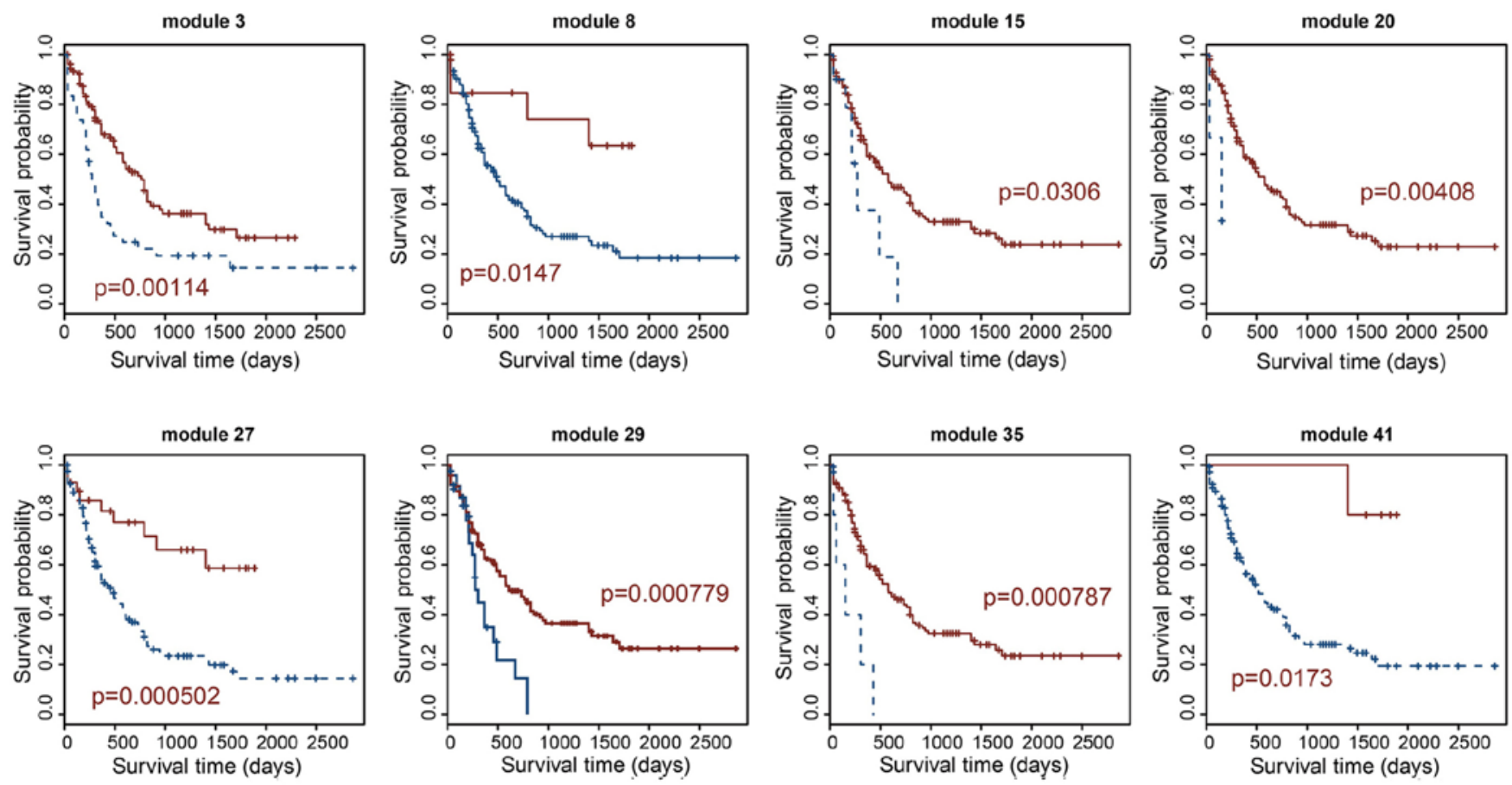

Figure 3. Kaplan-Meier survival analysis for each prognosis-related co-expression module of acute myeloid leukemia (AML). 
Table I. Information of lncRNA co-expression modules.

\begin{tabular}{|c|c|c|c|}
\hline $\begin{array}{l}\text { Module } \\
\text { name }\end{array}$ & $\begin{array}{l}\text { IncRNA } \\
\text { no. }\end{array}$ & P-value & IncRNAs \\
\hline 27 & 17 & 0.000502 & $\begin{array}{l}\text { BX284650.1.1; BX284650.2.1; BX248398.1.1; AL592284.1.1; RP11-495P10.2.1; RP11-277L2.2.1; } \\
\text { RP11-21M7.1.1; AC009948.5.1; RP11-519M16.1.1; RP11-361F15.2.1; AC073133.1.1; } \\
\text { RP11-426A6.7.1; AE000661.37.1; RP11-529H20.6.1; RP11-168G16.1.1; CRNDE; AL353997.1 }\end{array}$ \\
\hline 29 & 16 & 0.000779 & $\begin{array}{l}\text { RP11-430C7.4.1; AC074093.1.1; RP11-234O6.2.1; CTD-2324F15.2.1; RP11-492A10.1.1; } \\
\text { ABHD11-AS1; RP11-1057N3.1.1; CTC-774J1.2.1; RP11-444D3.1.1; LINC00477; RP11-24M17.7.1; } \\
\text { CTD-2335A18.1.1; RP11-61A14.1.1; RP11-960L18.1.1; RP11-486L19.2.1; RP5-1069P2.4.1 }\end{array}$ \\
\hline 35 & 9 & 0.000787 & $\begin{array}{l}\text { AC096579.13.1; AC096579.7.1; AC096670.3.1; RP11-133O22.6.1; RP11-492E3.2.1; } \\
\text { SFTA1P; AL928742.12.1; RP11-731F5.2.1; AL928768.3.1 }\end{array}$ \\
\hline 3 & 88 & 0.00114 & $\begin{array}{l}\text { RP11-206L10.11.1; RP11-181G12.2.1; RP11-576I22.2.1; RP11-145A3.1.1; AC104695.3.1; } \\
\text { AC013271.3.1; AC013268.5.1; RP11-1223D19.1.1; AC112229.7.1; ENSG00000273471; } \\
\text { RP13-1039J1.2.1; AC009299.3.1; RP11-446H18.3.1; SMARCA5-AS1; RP11-1336O20.2.1; } \\
\text { AC091878.1.1; ENSG00000273345; RP1-90J20.7.1; RP11-553A21.3.1; RP11-162J8.2.1; } \\
\text { RP11-351J23.1.1; AC005027.4.1; RP11-875O11.2.1; RP11-177H13.2.1; CTD-3107M8.4.1; } \\
\text { RP11-68L18.1.1; HAS2-AS1; RP11-273G15.2.1; RP11-498P14.5.1; RP11-308N19.4.1; } \\
\text { ZNF883; RP11-203J24.9.1; RP11-499P20.2.1; RP11-80K21.1.1; RP11-106M7.1.1; } \\
\text { RP11-326C3.11.1; AC104389.28.1; AC015691.13.1; SPON1; SHANK2-AS1; RP11-356J5.12.1; } \\
\text { RP11-598F7.4.1; CACNA1C-AS1; RP11-436I9.2.1; RP11-118B22.2.1; RP11-495K9.3.1; } \\
\text { RP11-394A14.2.1; LINC00355; MIR4500HG; RP11-44N21.4.1; RP11-941F15.1.1; WASIR2; } \\
\text { RP11-314O13.1.1; AC021593.1; AC105337.1; AC127496.3; ENSG00000266149; } \\
\text { ENSG00000265257; ENSG00000265485; CTD-2666L21.1.1; ENSG00000267147; } \\
\text { ENSG00000269110; AC092296.1.1; AC092295.7.1; AC012309.5.1; ENSG00000267470; } \\
\text { ENSG00000267640; AC016582.2.1; ENSG0000268262; AC011497.1; ENSG00000267058; } \\
\text { ENSG00000267188; CEACAM20; AC011450.1; ENSG00000269959; LINC00085; } \\
\text { ENSG00000267827; ENSG00000267454; AC004696.1; RP4-694B14.5.1; C20orf203; } \\
\text { AF127936.7.1; LINC00319; LINC00313; LL22NC03-86G7.1.1; RP4-756G23.5.1; } \\
\text { Z83851.3.1; LL0XNC01-116E7.2.1 }\end{array}$ \\
\hline 15 & 35 & 0.00408 & $\begin{array}{l}\text { RP5-1071N3.1.1; AC087590.3.1; RP11-81N13.1.1; RP11-553L6.2.1; RP11-18H21.1.1; } \\
\text { LINC00243; LINC00336; AC002480.3.1; NEFL; RP11-622O11.2.1; RP11-234A3.1.1; } \\
\text { RP11-229P13.19.1; RP13-25N22.1.1; RP11-730K11.1.1; RP11-627G23.1.1; RP11-493L12.2.1; } \\
\text { ENSG00000266923; RP11-173C20.1.1; RP11-90M5.1.1; LINC00402; RP11-58E21.3.1; } \\
\text { ENSG00000273065; CTD-2506J14.1.1; RP11-23P13.6.1; ENSG00000266088; ENSG00000267128; } \\
\text { RP11-77C3.3.1; LINC00494; RP4-669H2.1.1; LINC00161; MIAT; CTA-373H7.7.1; } \\
\text { RP11-265P11.2.1; DGKK; RP3-527F8.2.1 }\end{array}$ \\
\hline 8 & 54 & 0.0147 & $\begin{array}{l}\text { TTLL10-AS1; RP11-12L8.1.1; AC011893.3.1; AC005042.4.1; RP11-584P21.2.1; RP11-422J15.1.1; } \\
\text { RP11-121L11.1.1; AC034220.3.1; ENSG0000273299; XKR5; LINC00051; FAM201A; } \\
\text { RP11-195E11.3.1; ENSG00000268364; RP11-154D17.1.1; RP11-492E3.1.1; RP11-144G6.12.1; } \\
\text { ENSG00000270119; RP11-464F9.1.1; ENSG00000271816; ENSG00000272140; ENSG00000271880; } \\
\text { AGAP11; ENSG00000272508; RP11-399L7.2.1; RP11-554A11.6.1; RP11-166D19.1.1; } \\
\text { RP11-820L6.1.1; RP11-713P17.3.1; C14orf167; RP11-829H16.3.1; RP11-193F5.1.1;RP4-647C14.2.1; } \\
\text { LINC00341; RP11-37C7.1.1; RP11-358M11.2.1; RP11-809H16.2.1; ENSG00000272298; } \\
\text { AC091172.1; ENSG00000261924; CHST9-AS1; ENSG00000267013; ENSG00000267629; } \\
\text { ENSG00000267339; FXYD1; RP4-779E11.3.1; ENSG00000270408; RP4-568F9.6.1; RP4-705O1.1.1; } \\
\text { AL592528.1.1; RP1-274L7.1.1; AC004383.4.1; AC004383.5.1; RP1-260J9.2.1 }\end{array}$ \\
\hline 41 & 7 & 0.0173 & AC113607.1.1; RP11-356I2.4.1; RP11-504G3.1.1; AP003025.2.1; MEG3; MEG8; AL132709.7.1 \\
\hline 20 & 22 & 0.0306 & $\begin{array}{l}\text { RP11-98D18.9.1; RP11-466F5.8.1; AC007246.3.1; AC010127.3.1; ENSG00000271270; } \\
\text { CTD-2049J23.2.1; RP11-497D6.4.1; AC091729.9.1; RP4-555L14.5.1; RP11-539E17.4.1; } \\
\text { RP11-143M1.3.1; ENSG00000271086; RP11-554F20.1.1; LINC00202; RP11-887P2.1.1; } \\
\text { DIO3OS; ENSG00000262188; LINC00470; RP11-795F19.1.1; ENSG00000269696; } \\
\text { BX322557.10.1; AP001476.2.1 }\end{array}$ \\
\hline
\end{tabular}

lncRNA, long non-coding RNA. 


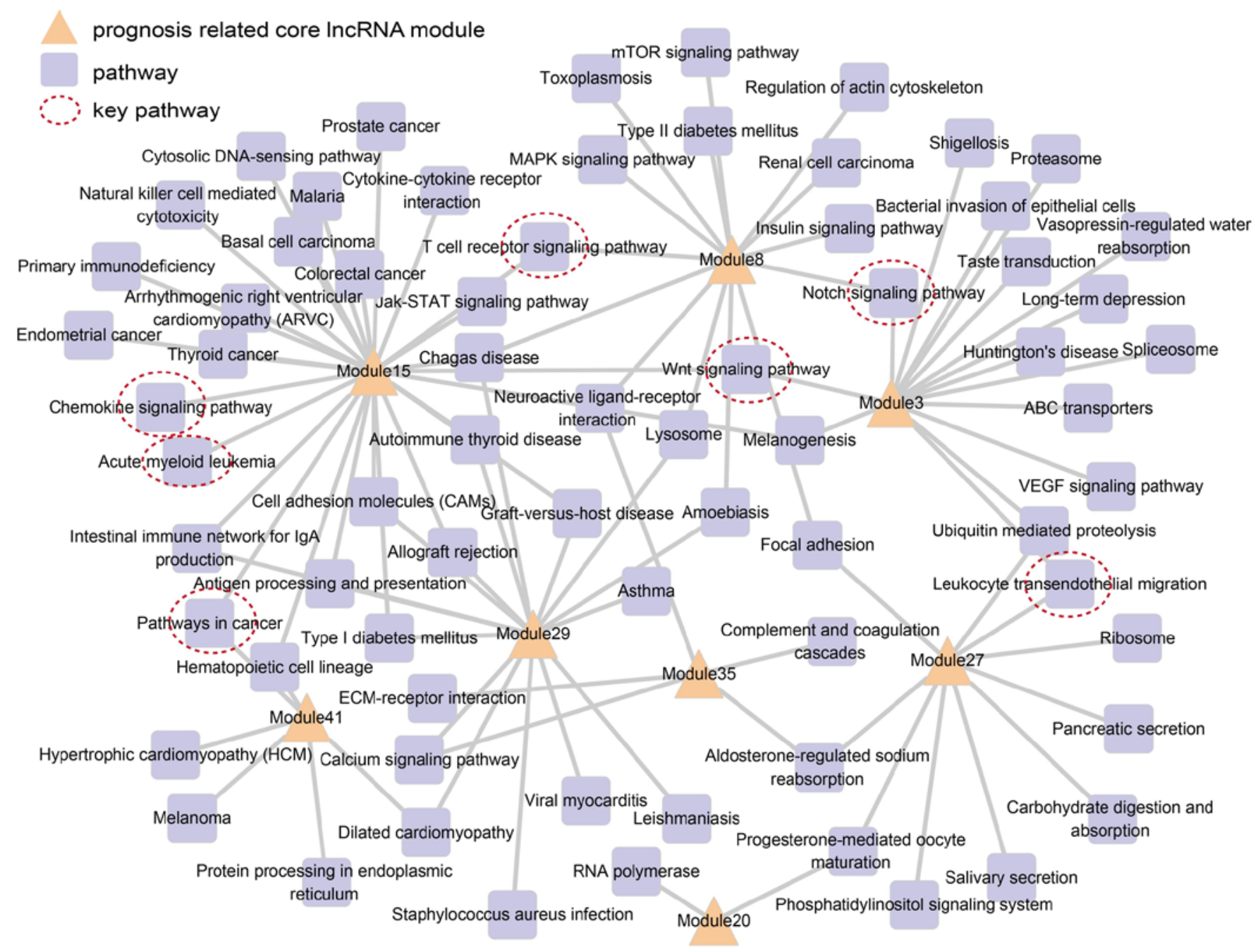

Figure 4. Prognosis-related module-pathway network of acute myeloid leukemia (AML). The triangles and rectangles represent nodes of modules and pathways, respectively.

a prognosis module pathway network was constructed based on these pathways, in which nodes represent IncRNA modules and pathways, and edges represent significantly enriched pathways (Fig. 4). Fig. 4 shows all prognosis-related lncRNA modules connected to more than one functional pathway. Among these, modules 15, 29 and 3 were involved in the most number of pathways, namely 27,16 and 15 , respectively. The most significant prognosis-related module, module 27, was involved in 10 pathways, including the leukocyte transendothelial migration pathway (23). This indicated that these prognosis lncRNA modules executed multiple biological functions. We also noted that some pathways were related with more than one lncRNA module, saling pathway (24), notch signaling pathway (25) and T cell receptor signaling pathway (26-28).

\section{Discussion}

In the present study, the expression levels of lncRNAs and mRNAs in AML were calculated, following which an LLCN was constructed (Fig. 2). This network contained 1,406 lncRNAs with 7,915 edges between them. The most connected lncRNA was RP11-492A10.1, which is an antisense RNA locating to chromosome 15 . To investigate the function of RP11-492A10.1, pathway enrichment analysis was performed on 125 protein-coding genes that co-expressed with RP11-492A10.1. The results revealed that these genes were involved in the neuroactive ligand-receptor interaction pathway, calcium signaling pathway, and chemokine signaling pathway. It has been demonstrated that CXCR4 chemokine receptor signaling induces the apoptosis of in AML cells via the regulation of the Bcl-2 family members, Bcl-xL, Noxa and Bak (22). This suggested that RP11-492A10.1 may be an important regulator in AML via co-expressed genes.

To identify prognosis-related lncRNA modules for AML, WGCNA was used to detect functional lncRNA co-expression modules. By integrating clinical survival data, 8 prognosisrelated IncRNA modules were identified from 42 IncRNA co-expression modules (Figs. 2 and 3). Modules 27 and 29 were the most significant prognosis-related lncRNA modules, which displayed the optimal performance in survival prediction ( $\mathrm{p}=0.000502$ and 0.000779 , respectively). Modules 27 and 29 consist of 17 and $16 \operatorname{lncRNAs}$, respectively, and the lncRNAs in them were highly connected within the modules, indicating that they interact closely with each other (Fig. 2). To further investigate the mechanisms of these prognosis-related lncRNA modules, functional pathway enrichment analysis 
A
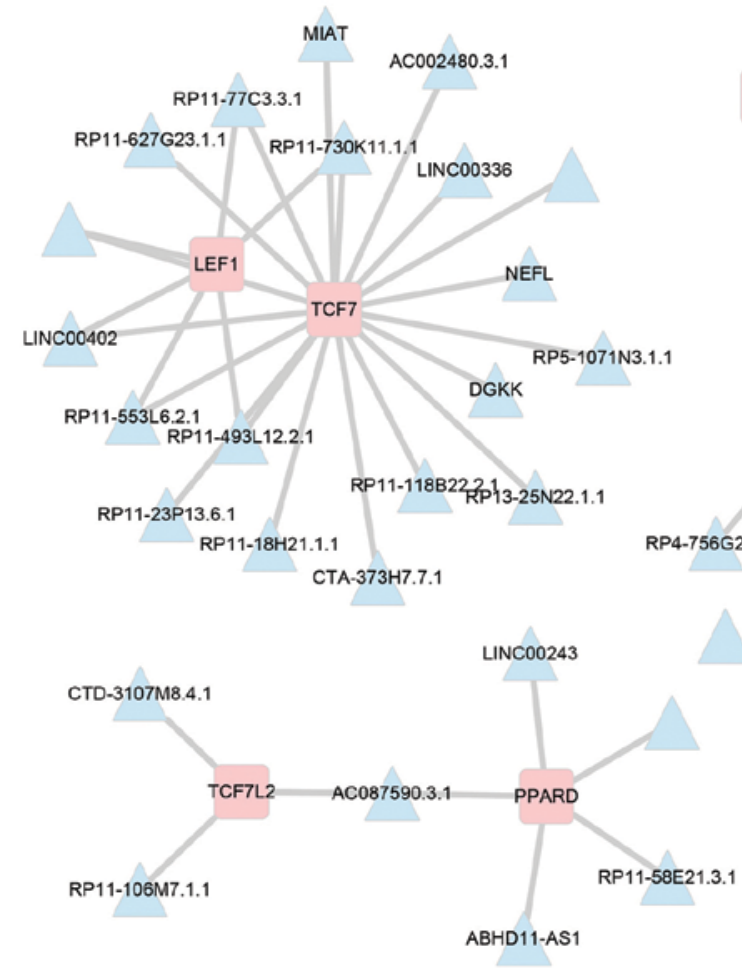

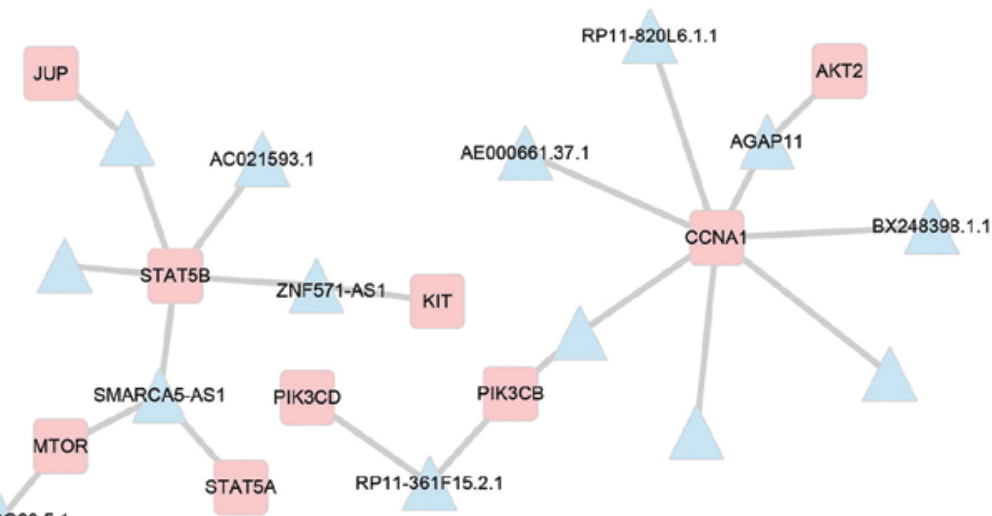
4-756G23.5.1
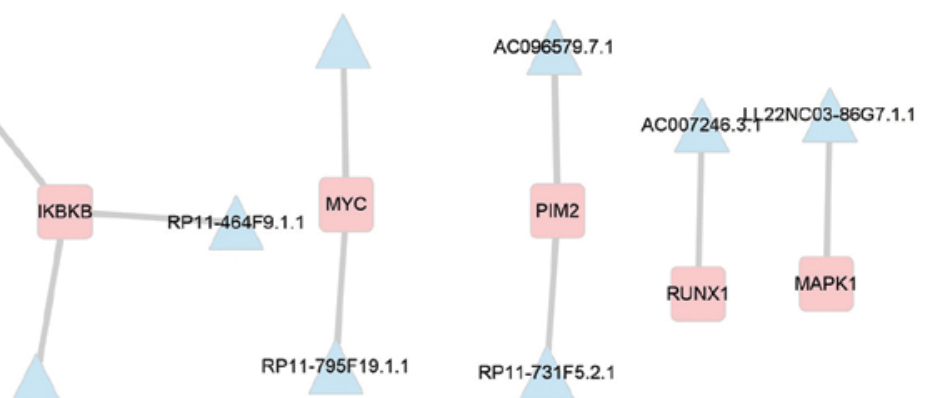

RP11-795F19.1.1 RP11-731F5.2.1

B

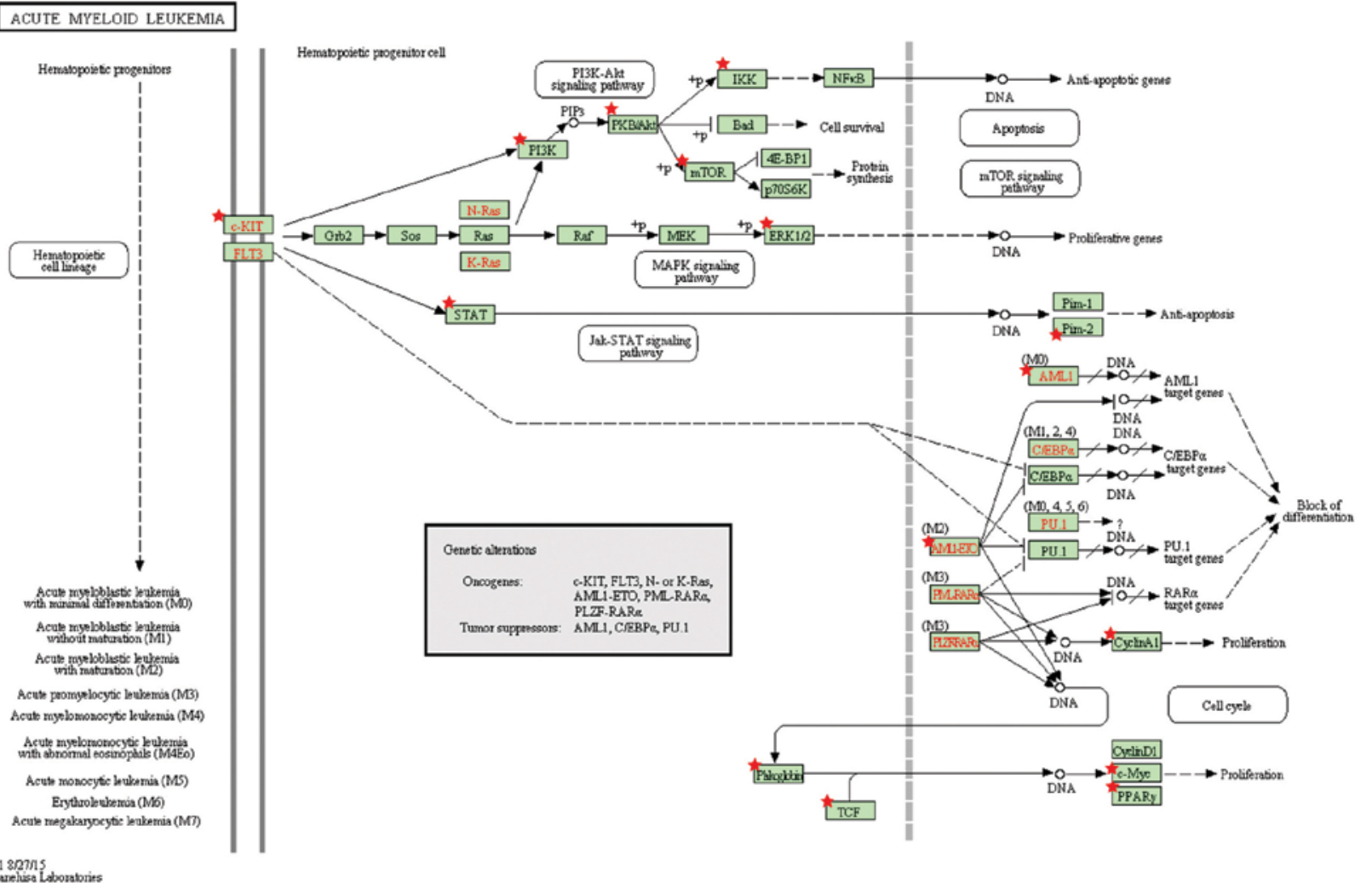

Figure 5. (A) Sub-network to illustrate potential long non-coding RNA (lncRNA)-mRNA co-expression associations in the acute myeloid leukemia (AML) pathway. The triangles and rectangles represent nodes of lncRNAs and mRNAs, respectively. (B) The AML pathway. Red stars indicate genes, which are potentially regulated by lncRNAs. 
was performed using co-expressed genes of lncRNAs in each prognostic lncRNA module and a prognostic lncRNA modulepathway network was constructed (Fig. 4). We noted that the acute myeloid leukemia pathway and pathway in cancer were identified. Besides this, the chemokine signaling pathway (22), leukocyte transendothelial migration pathway (23), WNT signaling pathway (24), notch signaling pathway (25), and $\mathrm{T}$ cell receptor signaling pathway (26-28) were found to be important in AML. Subsequently, to examine the detailed regulatory mechanisms of the prognostic modules, the co-expressed mRNAs of module 15 were mapped into the AML pathway (Fig. 5). As shown in Fig. 5A, there are 49 lncRNAs in prognosis modules co-expressed with 18 mRNAs involved in the acute myeloid leukemia pathway (Fig. 5B). The most connected genes lymphoid enhancer-binding factor 1 (LEF1) and transcription factor 7 (TCF7), both map on the TCF element in the acute myeloid leukemia pathway. Some studies have indicated that $L E F 1$ contributes to the pathophysiology of AML and may serve as a novel predictor for better treatment responses $(29,30)$. RP11-730K11.1.1 co-expressed with both LEF1 and TCF7, suggesting that it may play an important role in AML by regulating LEF1 and TCF7. ZNF571-AS1 correlated with both STAT5A and KIT. KIT is a crucial membrane protein, mutations of the KIT receptor tyrosine kinase are involved in the constitutive activation and development of AML and have a prognostic or possible therapeutic impact in AML (31). The constitutive activation of phospho-STAT5 was associated with a poor outcome in AML, this may be via the JAK/STAT signaling pathway (32). Thus, ZNF571-AS1 may be involved in AML via the JAK/STAT signaling pathway by regulating KIT and STAT5.

Although the results of the present study require further experimental verification, they provide possible prognostic markers for predicting patient outcome and provide further insight into the roles of IncRNAs in AML.

\section{Acknowledgements}

This study was supported by the Health and Family Planning Commission of Heilong jiang Province of China (no. 2014-322).

\section{References}

1. Döhner H, Estey EH, Amadori S, Appelbaum FR, Büchner T, Burnett AK, Dombret H, Fenaux P, Grimwade D, Larson RA, et al European LeukemiaNet: Diagnosis and management of acute myeloid leukemia in adults: Recommendations from an international expert panel, on behalf of the European LeukemiaNet. Blood 115: 453-474, 2010.

2. Schlenk RF, Döhner K, Krauter J, Fröhling S, Corbacioglu A, Bullinger L, Habdank M, Späth D, Morgan M, Benner A, et al German-Austrian Acute Myeloid Leukemia Study Group: Mutations and treatment outcome in cytogenetically normal acute myeloid leukemia. N Engl J Med 358: 1909-1918, 2008.

3. Marcucci G, Maharry KS, Metzeler KH, Volinia S, Wu YZ, Mrózek K, Nicolet D, Kohlschmidt J, Whitman SP, Mendler JH, et al: Clinical role of microRNAs in cytogenetically normal acute myeloid leukemia: miR-155 upregulation independently identifies high-risk patients. J Clin Oncol 31: 2086-2093, 2013.

4. Díaz-Beyá M, Navarro A, Ferrer G, Díaz T, Gel B, Camós M, Pratcorona M, Torrebadell M, Rozman M, Colomer D, et al: Acute myeloid leukemia with translocation $(8 ; 16)(\mathrm{p} 11 ; \mathrm{p} 13)$ and MYST3CREBBP rearrangement harbors a distinctive microRNA signature targeting RET proto-oncogene. Leukemia 27: 595-603, 2013.
5. Díaz-Beyá M, Brunet S, Nomdedéu J, Tejero R, Díaz T, Pratcorona M, Tormo M, Ribera JM, Escoda L, Duarte R, et al; Cooperative AML group CETLAM (Grupo Cooperativo Para el Estudio y Tratamiento de las Leucemias Agudas y Mielodisplasias): MicroRNA expression at diagnosis adds relevant prognostic information to molecular categorization in patients with intermediate-risk cytogenetic acute myeloid leukemia. Leukemia 28: 804-812, 2014.

6. Schwind S, Maharry K, Radmacher MD, Mrózek K, Holland KB, Margeson D, Whitman SP, Hickey C, Becker H, Metzeler KH, et al: Prognostic significance of expression of a single microRNA, miR-181a, in cytogenetically normal acute myeloid leukemia: A Cancer and Leukemia Group B study. J Clin Oncol 28: 5257-5264, 2010

7. Kapranov P, Cheng J, Dike S, Nix DA, Duttagupta R, Willingham AT, Stadler PF, Hertel J, Hackermüller J, Hofacker IL, et al: RNA maps reveal new RNA classes and a possible function for pervasive transcription. Science 316: 1484-1488, 2007.

8. Derrien T, Johnson R, Bussotti G, Tanzer A, Djebali S, Tilgner H, Guernec G, Martin D, Merkel A, Knowles DG, et al: The GENCODE v7 catalog of human long noncoding RNAs: Analysis of their gene structure, evolution, and expression. Genome Res 22: 1775-1789, 2012.

9. Hirano T, Yoshikawa R, Harada H, Harada Y, Ishida A and Yamazaki T: Long noncoding RNA, CCDC26, controls myeloid leukemia cell growth through regulation of KIT expression. Mol Cancer 14: 90, 2015.

10. Xu J, Li CX, Li YS, Lv JY, Ma Y, Shao TT, Xu LD, Wang YY, Du L, Zhang YP, et al: MiRNA-miRNA synergistic network: Construction via co-regulating functional modules and disease miRNA topological features. Nucleic Acids Res 39: 825-836, 2011.

11. Yoshimura K, Okanoue T, Ebise H, Iwasaki T, Mizuno M, Shima T, Ichihara J, Yamazaki K: Identification of novel noninvasive markers for diagnosing nonalcoholic steatohepatitis and related fibrosis by data mining. Hepatology 63: 462-473, 2016.

12. Langfelder P and Horvath S: WGCNA: An R package for weighted correlation network analysis. BMC Bioinformatics 9: $559,2008$.

13. Ivliev AE, 't Hoen PA and Sergeeva MG: Coexpression network analysis identifies transcriptional modules related to proastrocytic differentiation and sprouty signaling in glioma. Cancer Res 70: 10060-10070, 2010.

14. Clarke C, Madden SF, Doolan P, Aherne ST, Joyce H, O'Driscoll L, Gallagher WM, Hennessy BT, Moriarty M, Crown J, et al: Correlating transcriptional networks to breast cancer survival: A large-scale coexpression analysis. Carcinogenesis 34: 2300-2308, 2013.

15. Li Y, Tang H, Sun Z, Bungum AO, Edell ES, Lingle WL, Stoddard SM, Zhang M, Jen J, Yang P and Wang L: Network-based approach identified cell cycle genes as predictor of overall survival in lung adenocarcinoma patients. Lung Cancer 80: 91-98, 2013.

16. Udyavar AR, Hoeksema MD, Clark JE, Zou Y, Tang Z, Li Z, Li M, Chen H, Statnikov A, Shyr Y, et al: Co-expression network analysis identifies Spleen Tyrosine Kinase (SYK) as a candidate oncogenic driver in a subset of small-cell lung cancer. BMC Syst Biol 7 (Suppl 5): S1, 2013.

17. Chou WC, Cheng AL, Brotto M and Chuang CY: Visual gene-network analysis reveals the cancer gene co-expression in human endometrial cancer. BMC Genomics 15: 300, 2014.

18. Liu R, Cheng Y, Yu J, Lv QL and Zhou HH: Identification and validation of gene module associated with lung cancer through coexpression network analysis. Gene 563: 56-62, 2015.

19. Liao Q, Liu C, Yuan X, Kang S, Miao R, Xiao H, Zhao G, Luo H, $\mathrm{Bu} \mathrm{D}$, Zhao H, et al: Large-scale prediction of long non-coding RNA functions in a coding-non-coding gene co-expression network. Nucleic Acids Res 39: 3864-3878, 2011.

20. Yu J, Yu J, Cordero KE, Johnson MD, Ghosh D, Rae JM, Chinnaiyan AM and Lippman ME: A transcriptional fingerprint of estrogen in human breast cancer predicts patient survival. Neoplasia 10: 79-88, 2008.

21. Huang W, Sherman BT and Lempicki RA: Systematic and integrative analysis of large gene lists using DAVID bioinformatics resources. Nat Protoc 4: 44-57, 2009.

22. Kremer KN, Peterson KL, Schneider PA, Meng XW, Dai H, Hess AD, Smith BD, Rodriguez-Ramirez C, Karp JE, Kaufmann SH and Hedin KE: CXCR4 chemokine receptor signaling induces apoptosis in acute myeloid leukemia cells via regulation of the Bcl-2 family members Bcl-XL, Noxa, and Bak. J Biol Chem 288: 22899-22914, 2013. 
23. Gallay N, Anani L, Lopez A, Colombat P, Binet C, Domenech J, Weksler BB, Malavasi F and Herault O: The role of platelet/endothelial cell adhesion molecule 1 (CD31) and CD38 antigens in marrow microenvironmental retention of acute myelogenous leukemia cells. Cancer Res 67: 8624-8632, 2007.

24. Staal FJT, Famili F, Garcia Perez L and Pike-Overzet K: Aberrant Wnt signaling in leukemia. Cancers (Basel) 8: 78, 2016.

25. Heidel FH, Arreba-Tutusaus P, Armstrong SA and Fischer T: Evolutionarily conserved signaling pathways: acting in the shadows of acute myelogenous leukemia's genetic diversity. Clin Cancer Res 21: 240-248, 2015

26. Li Y: Alterations in the expression pattern of TCR zeta chain in T cells from patients with hematological diseases. Hematology 13: 267-275, 2008

27. Le Dieu R, Taussig DC, Ramsay AG, Mitter R, Miraki-Moud F, Fatah R, Lee AM, Lister TA and Gribben JG: Peripheral blood $\mathrm{T}$ cells in acute myeloid leukemia (AML) patients at diagnosis have abnormal phenotype and genotype and form defective immune synapses with AML blasts. Blood 114: 3909-3916, 2009.

28. Shi L, Chen S, Lu Y, Wang X, Xu L, Zhang F, Yang L, Wu X, $\mathrm{Li} \mathrm{B}$ and Li Y: Changes in the MALT1-A20-NF- $\kappa \mathrm{B}$ expression pattern may be related to T cell dysfunction in AML. Cancer Cell Int 13: 37, 2013.
29. Fu Y, Zhu H, Wu W, Xu J, Chen T, Xu B, Qian S, Li J and Liu P: Clinical significance of lymphoid enhancer-binding factor 1 expression in acute myeloid leukemia. Leuk Lymphoma 55: 371-377, 2014.

30. Metzeler KH, Heilmeier B, Edmaier KE, Rawat VP, Dufour A Döhner K, Feuring-Buske M, Braess J, Spiekermann K, Büchner T, et al: High expression of lymphoid enhancer-binding factor-1 (LEF1) is a novel favorable prognostic factor in cytogenetically normal acute myeloid leukemia. Blood 120: 2118-2126, 2012.

31. Yohe S: Molecular genetic markers in acute myeloid leukemia. J Clin Med 4: 460-478, 2015.

32. Garg S, Shanmukhaiah C, Marathe S, Mishra P, Babu Rao V, Ghosh K and Madkaikar M: Differential antigen expression and aberrant signaling via PI3/AKT, MAP/ERK, JAK/STAT, and Wnt/beta catenin pathways in Lin-/CD38-/CD34 cells in acute myeloid leukemia. Eur J Haematol 96: 309-317, 2016. 